'Departamento

de Enfermedades

Respiratorias, Facultad

de Medicina, Pontificia

Universidad Católica de

Chile, Santiago, Chile.

${ }^{2}$ Servicio de Medicina

Interna, Hospital San Juan

de Dios, Universidad de

Chile.

3Programa de Virología

Instituto de Ciencias

Biomédicas, Facultad de

Medicina, Universidad de

Chile.

${ }^{a}$ Alumno de Sexto Año

de Medicina, Pontificia

Universidad Católica de

Chile

Financiamiento: Proyecto

Fondecyt No 1085268

Recibido el 16 de junio de

2011, aceptado el 21 de

septiembre de 2011

Correspondencia a:

Dr. Fernando Saldías

Peñafiel

Departamento de

Enfermedades Respiratorias

Pontificia Universidad

Católica de Chile

Marcoleta 350, Santiago,

Chile.

Teléfonos: (562) 6331541

(562) 3543242

Fax: (562) 6335255

E-mail: fsaldias@med.

puc.cl

\section{Etiología y biomarcadores de inflamación sistémica en las exacerbaciones leves a moderadas de la enfermedad pulmonar obstructiva crónica}

\author{
FERNANDO SALDÍAS P. ${ }^{1}$, ORLANDO DÍAZ P. ${ }^{1}$, JORGE DREYSE D. ${ }^{2}$, \\ ALDO GAGGERO B. ${ }^{3}$, CHRISTIAN SANDOVAL A. ${ }^{\text {a }}$, CARMEN LISBOA B. ${ }^{1}$
}

\section{Etiology and biomarkers of systemic inflammation in mild to moderate COPD exacerbations}

Background: The etiology of acute exacerbations of chronic obstructive pulmonary disease (COPD) is heterogeneous and still under discussion. Inflammation increases during exacerbation of COPD. The identification of inflammatory changes will increase our knowledge and potentially guide therapy. Aim: To identify which inflammatory parameters increase during COPD exacerbations compared to stable disease, and to compare bacterial and viral exacerbations. Material and Methods: In 85 COPD patients (45 males, mean age $68 \pm 8$ years, FEV, $46 \pm 17 \%$ of predicted) sputum, nasopharyngeal swabs and blood samples were collected to identify the causative organism, during a mild to moderate exacerbation. Serum ultrasensitive $C$ reactive protein (CRP), fibrinogen and interleukin 6 (IL 6), neutrophil and leukocyte counts were measured in stable conditions, during a COPD exacerbation, 15 and 30 days post exacerbation. Results: A total of 120 mild to moderate COPD exacerbations were included. In 74 (61.7\%), a microbial etiology could be identified, most commonly Mycoplasma pneumoniae (15.8\%), Rhinovirus (15\%), Haemophilus influenzae (14.2\%), Chlamydia pneumoniae (11.7\%), Streptococcus pneumoniae (5.8\%) and Gram negative bacilli (5.8\%). Serum CRP, fibrinogen and IL 6, and neutrophil and leukocyte counts significantly increased during exacerbation and recovered at 30 days post exacerbation. Compared to viral exacerbations, bacterial aggravations were associated with a systemic inflammation of higher magnitude. Conclusions: Biomarkers of systemic inflammation increase during mild to moderate COPD exacerbations. The increase in systemic inflammation seems to be limited to exacerbations caused by bacterial infections.

(Rev Med Chile 2012; 140: 10-18).

Key words: C-reactive protein; Inflammation; Pulmonary disease chronic obstructive.
L a enfermedad pulmonar obstructiva crónica (EPOC) es una enfermedad prevenible evolución progresiva, que se caracteriza por la presencia de obstrucción crónica al flujo aéreo, asociada a una reacción inflamatoria anómala de la vía aérea frente a partículas o gases irritantes ${ }^{1,2}$.
Esta condición afecta a una fracción importante de la población chilena según el estudio PLA$\mathrm{TINO}^{3}$, siendo los principales factores de riesgo el tabaquismo y la exposición a contaminantes ambientales ${ }^{4}$. Es la cuarta causa de muerte en los países desarrollados y se cree que su frecuencia y mortalidad aumentarán significativamente en 
los próximos años, debido a la persistencia del hábito tabáquico en los varones, a su incremento en las mujeres y a factores demográficos como el aumento de la esperanza de vida ${ }^{1,2,5}$.

La EPOC es considerada una enfermedad inflamatoria de las vías aéreas y el parénquima pulmonar, caracterizada por aumento de la cantidad de neutrófilos, macrófagos y linfocitos CD8 ${ }^{6}$. Existiría una relación entre la inflamación local y la gravedad de la limitación al flujo aéreo y la evolución de la enfermedad ${ }^{7}$. Además, se ha demostrado que la inflamación pulmonar se extiende a otros territorios tisulares fuera del sistema respiratorio ${ }^{8}$.

La enfermedad tiene un curso crónico, lentamente progresivo, que puede conducir a la incapacidad e incluso a la muerte9. Sobre este curso crónico aparecen episodios agudos de exacerbación, con un aumento de los síntomas habituales (disnea y limitación de la actividad física). Las exacerbaciones constituyen un problema frecuente en los pacientes con EPOC y se han asociado a disminución de la capacidad funcional, declinación acelerada de la función pulmonar, menoscabo del estado de salud y calidad de vida, siendo responsables de muchas admisiones hospitalarias, y de este modo incrementarían los costos de la atención de salud y el riesgo de muerte ${ }^{10-15}$. Las infecciones bacterianas y virales son la principal causa de las exacerbaciones de la EPOC, seguidas de la pobre adherencia al tratamiento, la contaminación ambiental, embolia pulmonar y otras causas menos prevalentes ${ }^{16}$.

La EPOC se ha asociado a un estado de inflamación sistémica con aumento de varios biomarcadores séricos, tales como la proteína $\mathrm{C}$ reactiva (PCR), interleuquina 6 (IL6) y factor de necrosis tumoral alfa (TNF $\alpha)$; los cuales no serían buenos predictores del riesgo de exacerbación ${ }^{17-20}$.

La mayoría de los estudios de etiología y evaluación de los marcadores de inflamación sistémica han sido realizados en pacientes hospitalizados por exacerbaciones graves de la EPOC ${ }^{21-26}$. Sin embargo, gran parte de las exacerbaciones son manejadas en el ámbito ambulatorio debido a su bajo riesgo de complicaciones y muerte ${ }^{1,2}$. Por lo tanto, el propósito de nuestro estudio fue describir el cuadro clínico, la etiología y los cambios de algunos biomarcadores séricos de inflamación sistémica en las exacerbaciones infecciosas de la EPOC leves y moderadas de manejo ambulatorio.

\section{Pacientes y Métodos}

En una cohorte de seguimiento a tres años (enero de 2008 a diciembre de 2010) se evaluaron 85 pacientes ex-fumadores portadores de EPOC tabáquica definida de acuerdo a los criterios de $\mathrm{GOLD}^{1}$, en condiciones estables de su enfermedad y durante el primer o segundo episodio de exacerbación de manejo ambulatorio, quienes aceptaron participar en el protocolo firmando un acta de consentimiento informado aprobada por el comité de ética de nuestra institución. Los criterios de inclusión fueron ser mayores de 40 años, tener una historia de tabaquismo de 10 o más paquetes año, haber dejado de fumar por lo menos 6 meses antes y no tener alguna enfermedad que les impidiera realizar ejercicio físico o que por su gravedad no pudiera completar el período de seguimiento. Se excluyó a los pacientes con historia de asma, portadores de bronquiectasias, secuelas de tuberculosis, enfermedad neoplásica conocida o enfermedades caracterizadas por inflamación sistémica como las enfermedades colágeno vasculares.

Las exacerbaciones fueron definidas según criterios clínicos ${ }^{27}$ y el uso de recursos sanitarios, de acuerdo a la definición de Burge y Wedzicha ${ }^{28}$. Desde un punto de vista operacional, se consideró exacerbación leve a aquella tratada en el ámbito ambulatorio que requirió aumentar la terapia broncodilatadora con o sin uso de antibióticos, y exacerbación moderada a aquella que requirió tratamiento con antibióticos y corticoides sistémicos.

\section{Evaluación microbiológica}

Se realizó una evaluación microbiológica estandarizada, la cual incluyó los siguientes exámenes:

a) Muestras de expectoración para tinción de Gram y cultivo aeróbico corriente. En los pacientes que no expectoraban espontáneamente $(10 \%$ de los casos) se obtuvo muestras de esputo inducido mediante nebulización de solución salina al 3\% con nebulizador ultrasónico.

b) Muestras pareadas de sangre para serología (fase aguda y a los 30 días) de Mycoplasma pneumoniae (IgM por inmunofluorescencia indirecta e Ig G por técnica de ELISA; Zeus Scientific Inc; Raritan, NJ), Chlamydia pneumoniae y Chlamydia psittaci (IgM e IgG por técnica de microinmunofluorescencia; Vircell Inc; Granada, España).

c) Muestras de hisopado nasofaríngeo para la 
búsqueda de los virus influenza A y B, parainfluenza 1,2 y 3 , adenovirus, virus sincicial respiratorio y metapneumovirus.

d) Muestras de expectoración para detección de Rhinovirus humano (HRV) mediante una técnica de transcripción reversa acoplada a la reacción de polimerasa en cadena (RT-PCR), procesadas en el Programa de Virología de la Facultad de Medicina de la Universidad de Chile.

\section{Criterios de diagnóstico etiológico}

a) Aislamiento de un patógeno respiratorio en el cultivo de expectoración concordante con la tinción de Gram. Se consideró que la muestra era de buena calidad cuando tenía menos de 10 células epiteliales y más de 25 leucocitos por campo de menor aumento y sólo éstas se sembraron para cultivo.

b) Título de inmunoglobulina $\mathrm{M}$ sérica $\geq$ $1 / 10$ en muestra aislada o aumento $\geq 4$ veces del título de IgG (período agudo y convalecencia) para Chlamydia pneumoniae, Chlamydia psittaci y Mycoplasma pneumoniae.

c) Inmunofluorescencia directa positiva para influenza A y B, parainfluenza 1, 2 y 3 , adenovirus, virus sincicial respiratorio y metapneumovirus humano en hisopado nasofaríngeo.

d) Detección de Rhinovirus en muestra de expectoración por técnica de RT-PCR.

\section{Evaluación de la inflamación sistémica}

En un subgrupo de pacientes (n: 70) se midieron los biomarcadores de inflamación sistémica durante la fase estable de la enfermedad, al comienzo de la exacerbación, a los 15 y 30 días después de la exacerbación. Se midió la proteína C reactiva ultrasensible (PCRus) utilizando el método de inmunoensayo por nefelometría reforzado con partículas de látex (BN ProSpec analyzer, Dade-Behring ${ }^{\circledR}$, Deerfield, IL). El nivel mínimo pesquisable fue $0,175 \mathrm{mg} / \mathrm{L}$ y el rango varió entre 0,175 y $1.100 \mathrm{mg} / \mathrm{L}$. La interleuquina 6 sérica se determinó con el método de ELISA (Quantikine high sensitive ELISA R\&D Systems, Minneapolis, $\mathrm{MN}$ ) con límite inferior de detección de $0,039 \mathrm{pg} /$ $\mathrm{mL}$. El fibrinógeno sérico se determinó empleando un analizador de coagulación con el método coagulométrico de Clauss ${ }^{29}$ en un equipo ACL 9000. El recuento absoluto de neutrófilos y linfocitos se realizó en un frotis sanguíneo con recuento celular diferencial.

\section{Evaluación clínica y funcional}

En todos los pacientes incorporados al estudio, durante la fase estable de la enfermedad, se consignó las características sociodemográficas, las comorbilidades, se evaluó la magnitud de la disnea utilizando la escala modificada del Medical Research Council del Reino Unido ${ }^{30}$ (mMRC), se registró el número de exacerbaciones en los últimos 12 meses y se realizó una espirometría antes y después de la administración de salbutamol, siguiendo las normas de las Sociedades Americana de Tórax (ATS) y Europea de Enfermedades Respiratorias (ERS) ${ }^{31}$. Los valores se expresaron utilizando los valores de referencia de Hankinson $\mathrm{y} \operatorname{cols}^{32}$.

Durante la exacerbación, se registró los síntomas y signos, se midió la $\mathrm{SaO}_{2}$, se tomaron las muestras microbiológicas y los marcadores sistémicos de inflamación antes mencionados y se prescribió el tratamiento correspondiente. Los pacientes fueron controlados clínicamente y con pruebas funcionales a los 15 y 30 días después de la exacerbación.

\section{Análisis estadístico}

Los resultados fueron expresados como valores promedio \pm desviación estándar para las variables medidas en escala numérica y en porcentaje para las medidas en escala nominal. Las variables cualitativas fueron comparadas mediante la prueba de $\chi^{2}$ y las variables continuas con la prueba $t$ de Student. Para examinar la posible relación entre los biomarcadores séricos se empleó el coeficiente de correlación de Pearson. Para ello se utilizaron los programas Epi-Info 6.0 (CDC, Atlanta) y SPSS 16 (SPSS Inc., Chicago).

\section{Resultados}

Se evaluaron 85 pacientes ex-fumadores portadores de EPOC durante la fase estable y una exacerbación leve (35\%) o moderada $(65 \%)$ de la enfermedad. En la Tabla 1 se describen las características sociodemográficas y clínicas de los pacientes. La edad promedio de los pacientes fue 68 \pm 8 años (rango: $51-85$ ), 53\% varones, $49 \%$ etapas III y IV de GOLD, 85\% habían recibido la vacuna antiinfluenza y $47 \%$ la vacuna antineumocócica.

En esta cohorte de seguimiento a tres años se evaluaron 120 episodios de exacerbación de la EPOC (Tabla 1). Las principales manifestaciones 
Etiología y marcadores de inflamación sistémica en EPOC - F. Saldías et al

clínicas durante la exacerbación fueron el aumento de la disnea (91\%), tos productiva (96\%), expectoración purulenta $(80 \%)$, sibilancias $(65 \%)$ y síntomas sugerentes de una infección viral: coriza $(63 \%)$, odinofagia $(37 \%)$ y mialgias $(22 \%)$.

Tabla 1. Características clínicas de los pacientes atendidos por una exacerbación leve o moderada de la Enfermedad Pulmonar Obstructiva Crónica

\begin{tabular}{|c|c|c|}
\hline Características & $\mathbf{n}$ & $\%$ \\
\hline $\mathrm{N}$ & 85 & 100 \\
\hline Edad (años) & $68,6 \pm 7,7$ & (R: 51-85) \\
\hline Género M-F & $45-40$ & $53-47$ \\
\hline $\operatorname{CVF}(m L)$ & $2.621 \pm 711$ & ( $80 \pm 16 \%$ teórico) \\
\hline $\operatorname{VEF}_{1}(\mathrm{~mL})$ & $1.108 \pm 443$ & (46 $\pm 17 \%$ teórico) \\
\hline $\mathrm{VEF}_{1} / \mathrm{CVF}(\%)$ & $42,4 \pm 12,1$ & (R: 22-67) \\
\hline GOLD I-II-III-IV & $6-37-29-13$ & $7-44-34-15$ \\
\hline $\begin{array}{l}\text { Vacuna } \\
\text { antiinfluenza }\end{array}$ & $73 / 85$ & 85,8 \\
\hline $\begin{array}{l}\text { Vacuna } \\
\text { antineumocócica }\end{array}$ & $40 / 85$ & 47,0 \\
\hline $\begin{array}{l}\text { Duración } \\
\text { síntomas (días) }\end{array}$ & $6,8 \pm 6,2$ & (R: 1-30) \\
\hline Disnea & $108 / 119$ & 90,8 \\
\hline Tos & $114 / 119$ & 95,8 \\
\hline Expectoración & $111 / 119$ & 93,3 \\
\hline Esputo purulento & $95 / 119$ & 79,8 \\
\hline Sibilancias & $78 / 119$ & 65,5 \\
\hline Congestión nasal & $75 / 119$ & 63,0 \\
\hline Odinofagia & $44 / 119$ & 37,0 \\
\hline Sensación febril & $14 / 119$ & 11,8 \\
\hline Cefalea & 23/119 & 19,3 \\
\hline Mialgias & $26 / 119$ & 21,8 \\
\hline Pulso (lat/min) & $87,7 \pm 14,5$ & (R: 62-132) \\
\hline $\begin{array}{l}\text { PA Sistólica } \\
(\mathrm{mmHg})\end{array}$ & $129,6 \pm 17,7$ & (R: 100-180) \\
\hline $\begin{array}{l}\text { PA Diastólica } \\
(\mathrm{mmHg})\end{array}$ & $80,6 \pm 10,1$ & (R: 54-100) \\
\hline $\begin{array}{l}\text { Frecuencia respi- } \\
\text { ratoria (resp/min) }\end{array}$ & $21,3 \pm 5,1$ & (R: 12-40) \\
\hline Temperatura $\left({ }^{\circ} \mathrm{C}\right)$ & $36,2 \pm 0,4$ & (R: $35-38,4)$ \\
\hline $\mathrm{SaO}_{2}(\%)$ & $92,0 \pm 4,4$ & (R: 67-98) \\
\hline
\end{tabular}

Nota: Se evaluaron 120 episodios de exacerbación en una cohorte de seguimiento a tres años de 85 pacientes exfumadores con EPOC. M: Masculino, F. Femenino, CVF: Capacidad vital forzada, $V E F_{1}$ : Volumen espiratorio forzado del primer segundo, PA: Presión arterial, $\mathrm{SaO}_{2}$ : Saturación arterial de oxígeno.
Los principales microorganismos aislados fueron Mycoplasma pneumoniae (15,8\%), Rhinovirus (15\%), Haemophilus influenzae (14,2\%), Chlamydia pneumoniae (11,7\%), Streptococcus pneumoniae $(5,8 \%)$ y bacilos Gramnegativos aeróbicos $(5,8 \%)$, no se logró identificar el agente causal en $38,3 \%$ de los casos (Tablas 2 y 3 ). El cuadro clínico fue similar en los pacientes con exacerbaciones de origen viral, bacteriano o infección mixta. En la Tabla 4 se describe el rendimiento de los exámenes microbiológicos y en la Tabla 5 el tratamiento prescripto a los pacientes exacerbados. Dos tercios de los pacientes estaban siendo

Tabla 2. Etiología de las exacerbaciones infecciosas de la Enfermedad Pulmonar Obstructiva Crónica manejadas en el ámbito ambulatorio

\begin{tabular}{|lrr|}
\hline Microorganismos & n & \% \\
\hline Mycoplasma pneumoniae (Mp) & 19 & 15,8 \\
Haemophilus influenzae (Hi) & 17 & 14,2 \\
\hline Chlamydia pneumoniae (Cp) & 14 & 11,7 \\
Streptococcus pneumoniae (Sp) & 7 & 5,8 \\
\hline Pseudomonas aeruginosa (Pa) & 3 & 2,5 \\
Moraxella catarrhalis (Mc) & 1 & 0,8 \\
Klebsiella pneumoniae & 1 & 0,8 \\
\hline Escherichia coli (Ec) & 1 & 0,8 \\
\hline Enterobacter intermedius & 1 & 0,8 \\
Rhinovirus (Rh) & 18 & 15,0 \\
Virus sincicial respiratorio & 4 & 3,3 \\
Virus influenza B & 2 & 1,7 \\
Virus parainfluenza & 1 & 0,8 \\
\hline Metapneumovirus & 1 & 0,8 \\
\hline Desconocida & 46 & 38,3 \\
\hline
\end{tabular}

Nota: En 60 pacientes se aisló un microorganismo, en 12 pacientes dos microorganismos (Sp-Cp: 2; Mp-Rh: 2; MpCp: 2; Sp-Rh: 1; Rh-Hi: 1; Mc-Pa: 1; Hi-Cp: 1; Hi-Mp: 1; MpEc: 1) y en 2 pacientes tres microorganismos (Rh-Mp-Cp: 1; Hi-Rh-Cp: 1).

Tabla 3. Etiología de las exacerbaciones leve a moderadas de la Enfermedad Pulmonar Obstructiva Crónica manejadas en el ámbito ambulatorio

\begin{tabular}{|lrr|}
\hline Etiología & n & \% \\
\hline Virus respiratorios & 20 & 16,7 \\
\hline Bacterias & 48 & 40,0 \\
\hline Infección mixta (bacteria +virus) & 6 & 5,0 \\
\hline Desconocida & 46 & 38,3 \\
\hline
\end{tabular}


Tabla 4. Rendimiento diagnóstico de los exámenes microbiológicos en pacientes atendidos por una exacerbación leve a moderada de la Enfermedad Pulmonar Obstructiva Crónica

\begin{tabular}{|lcc|}
\hline Rendimiento diagnóstico & n & \% \\
\hline Gram y cultivo de expectoración & $30 / 93$ & 32,2 \\
\hline Serología de Mycoplasma pneumoniae & $19 / 105$ & 18,1 \\
\hline $\begin{array}{lrr}\text { Serología de Chlamydia pneumoniae } \\
\text { Inmunofluorescencia de virus respira- }\end{array}$ & $14 / 101$ & 13,9 \\
torios & & 7,0 \\
RT-PCR de Rhinovirus & $18 / 106$ & 17,0 \\
\hline
\end{tabular}

Tabla 5. Tratamiento de los pacientes atendidos por exacerbaciones infecciosas de la Enfermedad Pulmonar Obstructiva Crónica en el ámbito ambulatorio

\begin{tabular}{|lcc|}
\hline Tratamiento & n & \% \\
\hline$\beta_{2}$-adrenérgicos de acción corta & 111 & 92,5 \\
\hline Anticolinérgico de acción corta & 56 & 46,7 \\
\hline$\beta_{2}$-adrenérgicos de acción larga & 78 & 65,0 \\
\hline Anticolinérgico de acción larga & 54 & 45,0 \\
\hline Corticoides inhalados & 80 & 66,7 \\
Corticoides sistémicos & 78 & 65,0 \\
\hline Teofilinas & 13 & 10,8 \\
\hline Antibióticos & 106 & 88,3 \\
\hline Oxígenoterapia & 11 & 9,2 \\
\hline
\end{tabular}

Nota: Los principales antibióticos prescriptos fueron agentes $\beta$-lactámicos (n: 13), macrólidos (n: 19) y fluoroquinolonas (n: 74). tratados con agonistas $\beta 2$ adrenérgicos de acción larga y corticoides inhalatorios en dosis bajas, y el $45 \%$ con anticolinérgicos de acción larga.

En el subgrupo que se midieron los marcadores de inflamación sistémica (PCRus, fibrinógeno e IL 6 séricos, recuento de leucocitos y neutrófilos), estos se elevaron significativamente durante la exacerbación y tendieron a retornar a sus valores basales a los 30 días (Tabla 6). Este aumento de los marcadores inflamatorios se observó en las infecciones bacterianas, pero no en las infecciones virales (Figura 1). Los cambios en los niveles séricos de IL 6 se correlacionaron moderadamente con los valores de PCR ultrasensible (Figura 2).

\section{Discusión}

Los hallazgos más importantes del presente estudio fueron: 1) Los principales microorganismos aislados en las exacerbaciones leve y moderadas de la EPOC de manejo ambulatorio fueron $\mathrm{Myco-}$ plasma pneumoniae, Rhinovirus, Haemophilus influenzae, Chlamydia pneumoniae y Streptococcus pneumoniae; 2) Los virus respiratorios fueron aislados en $22 \%$ y las bacterias clásicas en $45 \%$ de los pacientes exacerbados; 3) El cuadro clínico de la exacerbación no permitió predecir el agente causal; 4) Los biomarcadores de inflamación sistémica (PCRus, fibrinógeno e IL 6 séricos, recuento de leucocitos y neutrófilos) se elevaron en las exacerbaciones infecciosas de origen bacteriano.

Las infecciones bacterianas y virales, la contaminación ambiental y la pobre adherencia al tratamiento constituyen las principales causas de

Tabla 6. Evolución de los biomarcadores de inflamación sistémica en $\mathbf{7 0}$ pacientes atendidos por exacerbaciones infecciosas de la Enfermedad Pulmonar Obstructiva Crónica en el ámbito ambulatorio

\begin{tabular}{|c|c|c|c|c|c|}
\hline $\begin{array}{l}\text { Biomarcadores de } \\
\text { inflamación sistémica }\end{array}$ & $\begin{array}{c}\text { Basal } \\
(\bar{x} \pm D E)\end{array}$ & 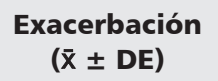 & $\begin{array}{l}15 \text { días } \\
(\bar{x} \pm D E)\end{array}$ & $\begin{array}{l}30 \text { días } \\
(\bar{x} \pm D E)\end{array}$ & $\begin{array}{c}p \\
\text { (ANOVA) }\end{array}$ \\
\hline Proteína C reactiva (mg/L) & $3,74 \pm 4,32$ & $27,30 \pm 42,75^{*}$ & $9,33 \pm 10,80$ & $6,66 \pm 8,86$ & $<0,0001$ \\
\hline Fibrinógeno (mg/dL) & $319,9 \pm 57,1$ & $395,2 \pm 104,1^{*}$ & $351,2 \pm 73,8$ & $337,9 \pm 69,6$ & 0,0002 \\
\hline Leucocitos $\left(\mathrm{x} \mathrm{mm}^{3}\right)$ & $7.205 \pm 1.525$ & $8.919 \pm 3.721^{*}$ & $8.138 \pm 3.054$ & $7.383 \pm 1.805$ & 0,015 \\
\hline Neutrófilos (x mm³) & $4.687 \pm 1.385$ & $6.302 \pm 3.800^{*}$ & $5.505 \pm 2.650$ & $4.888 \pm 1.883$ & 0,022 \\
\hline Linfocitos $\left(x \mathrm{~mm}^{3}\right)$ & $1.733 \pm 533$ & $1.667 \pm 695$ & $1.714 \pm 671$ & $1.667 \pm 591$ & 0,9478 \\
\hline Interleuquina 6 (pg/mL) & $3,81 \pm 3,27$ & $13,18 \pm 14,07^{*}$ & $7,53 \pm 14,36$ & $4,46 \pm 6,01$ & 0,002 \\
\hline
\end{tabular}

*Los niveles séricos de proteína $C$ reactiva ultrasensible, fibrinógeno e interleuquina 6 y el recuento de leucocitos y neutrófilos aumentaron significativamente durante la exacerbación comparado con la condición basal. 


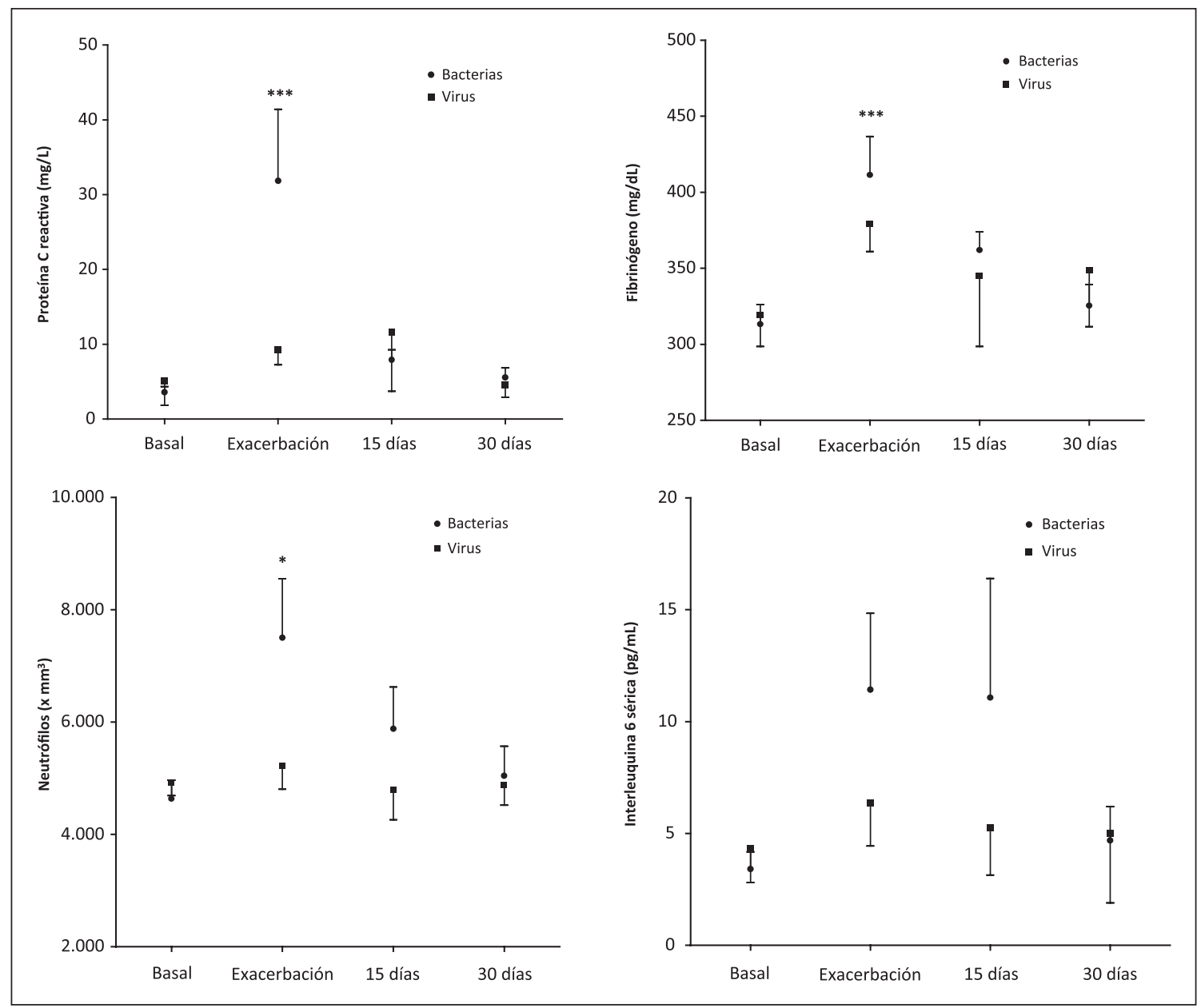

Figura 1. Evolución de los biomarcadores de inflamación sistémica en pacientes atendidos por exacerbaciones infecciosas virales y bacterianas de la Enfermedad Pulmonar Obstructiva Crónica. * $p<0,05$ y *** $p<0,001$ vs condición basal.

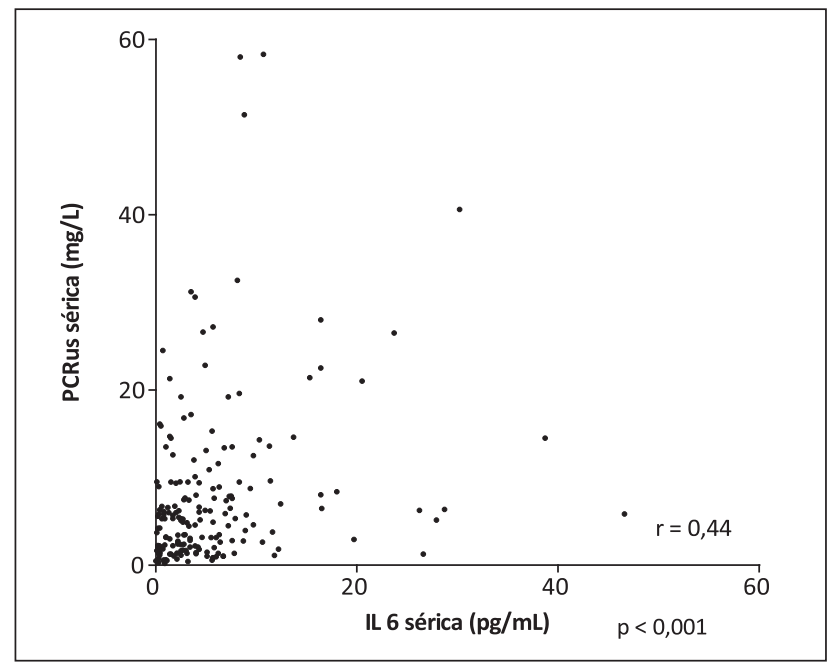

Figura 2. Correlación entre los niveles séricos de interleuquina 6 (IL 6) y proteína C reactiva ultrasensible (PCRus) en pacientes atendidos por exacerbaciones infecciosas de la Enfermedad Pulmonar Obstructiva Crónica. 
las exacerbaciones de la EPOC $1,2,8-13$. Similar a lo descrito en otros estudios ${ }^{1,14,22-27}$, los principales microorganismos involucrados en las exacerbaciones infecciosas de la EPOC fueron Rhinovirus, Haemophilus influenzae, bacterias atípicas, Streptococcus pneumoniae, bacilos Gramnegativos, virus influenza, parainfluenza y sincicial respiratorio. El cuadro clínico fue similar en las infecciones bacterianas, virales y de etiología desconocida. Las infecciones bacterianas se han asociado a esputo purulento y aumento de los neutrófilos en la vía aérea, retornando a la condición basal con el tratamiento antibiótico ${ }^{33}$. Sin embargo, el tratamiento antibiótico ha demostrado ser beneficioso sólo en el manejo de las exacerbaciones graves, especialmente en pacientes con EPOC avanzado y múltiples síntomas de exacerbación ${ }^{34}$.

Las infecciones virales de la vía aérea superior pueden precipitar exacerbaciones de la EPOC, especialmente Rhinovirus, virus sincicial respiratorio y virus influenza ${ }^{35}$. El 60\% de las exacerbaciones de la EPOC se asocian a síntomas de resfrío común, tales como congestión nasal, coriza y odinofagia $^{36}$, los cuales estuvieron presentes en nuestros enfermos y explicarían la alta tasa de detección de Rhinovirus. Se ha planteado que las exacerbaciones virales serían de mayor gravedad y más prolongadas, afectando negativamente la calidad de vida de los enfermos ${ }^{37}$. Los pacientes con EPOC avanzado y exacerbaciones frecuentes tendrían mayor susceptibilidad a las infecciones virales por mecanismos sólo parcialmente esclarecidos. Uno de ellos sería el aumento de la expresión de la molécula de adhesión intercelular 1 (ICAM-1) en las células epiteliales de la vía aérea, el cual actúa como receptor de Rhinovirus, ocasionado por la infección viral mediante la activación del factor de transcripción nuclear kappa $\beta(\mathrm{NF} \mathrm{K} \beta)^{38}$. Los diferentes tipos de Rhinovirus pueden ser detectados con mayor facilidad de muestras de esputo que de la vía aérea superior, lo cual pone en evidencia su rol patogénico en las infecciones del tracto respiratorio inferior ${ }^{39}$. Las infecciones virales, al igual que las bacterianas, están asociadas a aumento del recuento de neutrófilos en el esputo, sin embargo, también aumenta el número de eosinófilos en la vía aérea, lo que sugiere la participación de otros mediadores de la inflamación ${ }^{25}$.

Se estima que $25 \%$ de los pacientes hospitalizados por una exacerbación grave tendrían una coinfección entre virus y bacterias, las cuales se asociarían a cuadros de mayor severidad y estadía hospitalaria más prolongada. Así, la infección concomitante de $H$. influenzae y Rhinovirus se ha asociado a mayor caída del $\mathrm{VEF}_{1}$ e incremento de CXCL8 en esputo e IL 6 sérica ${ }^{40}$. En las muestras obtenidas de pacientes ambulatorios encontramos coinfección en 5\% de los casos.

La inflamación es una condición patológica importante asociada a la EPOC ${ }^{41}$ y las manifestaciones clínicas de las exacerbaciones son atribuidas a un aumento de la inflamación local y sistémica gatilladas por las infecciones bacterianas o virales y estímulos no infecciosos tales como la contaminación ambiental ${ }^{14,17-20,35,36}$. Se observa un aumento en el recuento de neutrófilos, que explica el esputo purulento, y en la liberación de citoquinas proinflamatorias en la vía aérea (TNF $\alpha$, IL 6, IL 8, LT B $\left.)_{4}\right)^{14,42}$. Similar a lo descrito en nuestro estudio $^{17-20,35}$, varios biomarcadores de inflamación sistémica (PCRus, fibrinógeno, TNF $\alpha$, IL 6) que están discretamente elevados en pacientes con EPOC estable, se incrementan significativamente durante las exacerbaciones y tienden a retornar a la condición basal después de 2-4 semanas (Tabla 6). Se ha planteado que este incremento reflejaría la sobreproducción de citoquinas en el pulmón que pasarían a la circulación sistémica ${ }^{14,43}$. Hurst y cols $^{44}$ examinaron 36 biomarcadores séricos durante los episodios de exacerbación de 90 pacientes con EPOC, encontrando que la elevación de la proteína $\mathrm{C}$ reactiva sérica sería el biomarcador sistémico más selectivo, aunque inespecífico, presente durante una exacerbación. Este hallazgo concuerda con la elevación significativa de la PCRus reportada en nuestro estudio.

Sin embargo, la utilidad de los biomarcadores sistémicos para predecir el riesgo de desarrollar exacerbaciones no ha sido claramente establecida y no se correlaciona con su gravedad o pronósti$\mathrm{CO}^{14,17-20}$. En el estudio de Perera y cols ${ }^{45}$, la persistencia de niveles de PCR sérica elevados a las dos semanas de una exacerbación permitía predecir una nueva exacerbación, y Groenewegen y cols ${ }^{46}$ demostraron que el fibrinógeno sérico elevado es un factor de riesgo independiente de exacerbaciones en pacientes con EPOC.

En nuestro estudio, el aumento de los biomarcadores de inflamación sistémica (PCRus, fibrinógeno e IL 6 sérica, recuento de leucocitos y neutrófilos) estuvo limitado a las exacerbaciones de origen bacteriano, lo cual ya había sido descrito 
por Bathoorn y cols en una cohorte similar de pacientes con exacerbaciones leves y moderadas de la EPOC de manejo ambulatorio ${ }^{47}$. De hecho, se ha planteado el uso de biomarcadores sistémicos específicos de infección bacteriana que pudieran orientar a los médicos clínicos en el uso racional de los antimicrobianos.

En resumen, en este estudio describimos el cuadro clínico y los principales agentes virales y bacterianos involucrados en las exacerbaciones leves y moderadas de la EPOC de manejo ambulatorio en nuestro medio. Las infecciones bacterianas se asociaron a elevación significativa de los biomarcadores séricos, los cuales podrían ser considerados junto a la evaluación clínica en la prescripción racional de los antibióticos en los servicios de atención primaria.

\section{Referencias}

1. Global Initiative for Chronic Obstructive Lung Disease (GOLD). Global Strategy for the diagnosis, management and prevention of chronic obstructive pulmonary disease 2009. www.goldcopd.com

2. Peces-Barba G, Barberà JA, Agustí A, Casanova C, Casas A, Izquierdo JL, et al. Diagnosis and management of chronic obstructive pulmonary disease: joint guidelines of the Spanish Society of Pulmonology and Thoracic Surgery (SEPAR) and the Latin American Thoracic Society (ALAT). Arch Bronconeumol 2008; 44: 271-81.

3. Menezes AM, Pérez-Padilla R, Jardim JR, Muino A, López MV, Valdivia G, et al. Chronic obstructive pulmonary disease in five Latin American cities (the PLATINO study): a prevalence study. Lancet 2005; 366: 1875-81.

4. Jindal SK, Aggarwal AN, Chaudhry K, Chhabra SK, D'Souza GA, Gupta D, et al. A multicentric study on epidemiology of chronic obstructive pulmonary disease and its relationship with tobacco smoking and environmental tobacco smoke exposure. Indian J Chest Dis Allied Sci 2006; 48: 23-9.

5. Murray CJ, López AD. Alternative projections of mortality and disability by cause 1990-2020: Global Burden of Disease Study. Lancet 1997; 349: 1498-504.

6. Riise GC, Ahlstedt S, Larsson S, Enander I, Jones I, Larsson $\mathrm{P}$, et al. Bronchial inflammation in chronic bronchitis assessed by measurement of cell products in bronchial lavage fluid. Thorax 1995; 50: 360-5.

7. Crapo RO, Jensen RL, Hargreave FE. Airway inflammation in COPD: physiological outcome measures and induced sputum. Eur Respir J 2003; 41(Suppl): 19S-28S.

8. Barnes PJ. Chronic obstructive pulmonary disea- se: effects beyond the lungs. PLoS Med 2010; 7 (3): e1000220.

9. Mannino DM, Doherty DE, Buist S. Global Initiative on Obstructive Lung Disease (GOLD) classification of lung disease and mortality: findings from the Atherosclerosis Risk in Communities (ARIC) study. Respir Med 2006; 100: 115-22.

10. Connors AF Jr, Dawson NV, Thomas C, Harrell Fe Jr, Desbiens N, Fulkerson WJ, et al. Outcomes following acute exacerbation of severe chronic obstructive lung disease. The SUPPORT investigators (Study to Understand Prognoses and Preferences for Outcomes and Risks of Treatments). Am J Respir Crit Care Med 1996; 154: 959-67.

11. Seemungal TA, Donaldson GC, Paul EA, Bestall JC, Jeffries DJ, Wedzicha JA. Effect of exacerbation on quality of life in patients with chronic obstructive pulmonary disease. Am J Respir Crit Care Med 1998; 157: 1418-22.

12. Donaldson GC, Seemungal TA, Bhowmik A, Wedzicha JA. Relationship between exacerbation frequency and lung function decline in chronic obstructive pulmonary disease. Thorax 2002; 57: 847-52.

13. Miravitlles M, Murio C, Guerrero T, Gisbert R, on behalf of the DAFNE Study Group. Costs of chronic bronchitis and COPD: a 1-year follow-up study. Chest 2003; 123: 784-91.

14. Miravitlles M, Ferrer M, Pont A, Zalacain R, Álvarez-Sala JL, Masa JF, et al. Effect of exacerbations on quality of life in patients with chronic obstructive pulmonary disease: a 2 year follow up study. Thorax 2004; 59: 387-95.

15. Spencer S, Calverley PM, Burge PS, Jones PW. Impact of preventing exacerbations on deterioration of health status in COPD. Eur Respir J 2004; 23: 698-702.

16. Celli BR, Barnes PJ. Exacerbations of chronic obstructive pulmonary disease. Eur Respir J 2007; 29: 1224-38.

17. Wouters EF, Creutzberg EC, Schols AM. Systemic effects in COPD. Chest 2002; 121 (5 Suppl): 127S-130S.

18. Oudijk EJ, Lammers JW, Koenderman L. Systemic inflammation in chronic obstructive pulmonary disease. Eur Respir J 2003; 46 (Suppl): 5S-13S.

19. Agusti AG, Noguera A, Sauleda J, Sala E, Pons J, Busquets X. Systemic effects of chronic obstructive pulmonary disease. Eur Respir J 2003; 21: 347-60.

20. Gan WQ, Man SF, Senthilselvan A, Sin DD. Association between chronic obstructive pulmonary disease and systemic inflammation: a systematic review and a metaanalysis. Thorax 2004; 59: 574-80.

21. Lieberman D, Ben-Yaakov M, Lazarovich Z, Ohana B, Boldur I. Chlamydia pneumoniae infection in acute exacerbations of chronic obstructive pulmonary disease: analysis of 250 hospitalizations. Eur J Clin Microbiol 
Infect Dis 2001; 20: 698-704.

22. Rohde G, Wiethege A, Borg I, Kauth M, Bauer TT, Gillissen A, et al. Respiratory viruses in exacerbations of chronic obstructive pulmonary disease requiring hospitalisation: a case-control study. Thorax 2003; 58: 37-42.

23. Murphy TF. The role of bacteria in airway inflammation in exacerbations of chronic obstructive pulmonary disease. Curr Opin Infect Dis 2006; 19: 225-30.

24. Papi A, Bellettato CM, Braccioni F, Romagnoli M, Casolari P, Caramori G, et al. Infections and airway inflammation in chronic obstructive pulmonary disease severe exacerbations. Am J Respir Crit Care Med 2006; 173: 1114-21.

25. Roche N, Kouassi B, Rabbat A, Mounedji A, Lorut C, Huchon G. Yield of sputum microbiological examination in patients hospitalized for exacerbations of chronic obstructive pulmonary disease with purulent sputum. Respiration 2007; 74: 19-25.

26. Nseir S, Cavestri B, Di Pompeo C, Diarra M, Brisson H, Lemyze $\mathrm{M}$, et al. Factors predicting bacterial involvement in severe acute exacerbations of chronic obstructive pulmonary disease. Respiration 2008; 76: 253-60.

27. Anthonisen NR, Manfreda J, Warren CP, Hershfield ES, Harding GK, Nelson NA. Antibiotic therapy in exacerbations of chronic obstructive pulmonary disease. Ann Intern Med 1987; 106: 196-204.

28. Burge S, Wedzicha JA. COPD exacerbations: definitions and classifications. Eur Respir J 2003;41(Suppl): 46S$53 \mathrm{~S}$.

29. Clauss A. Gerinnungs physiologische schnell methode zur bestinnung des fibrinogens. Acta Haematol 1957; 17: 237-46.

30. Mahler DA, Wells CK. Evaluation of clinical methods for rating dyspnea. Chest 1988; 93: 580-6.

31. Miller MR, Hankinson J, Brusasco V, Burgos F, Casaburi $\mathrm{R}$, Coates A, et al. Standardisation of spirometry. Eur Respir J 2005; 26: 319-38.

32. Hankinson JL, Odencrantz JR, Fedan KB. Spirometric reference values from a sample of the general U.S. population. Am J Respir Crit Care Med 1999; 159: 179-87.

33. Patel IS, Seemungal TA, Wilks M, Lloyd-Owen SJ, Donaldson GC, Wedzicha JA. Relationship between bacterial colonisation and the frequency, character, and severity of COPD exacerbations. Thorax 2002; 57: 759-64.

34. Puhan MA, Vollenweider D, Latshang T, Steurer J, Steurer-Stey C. Exacerbations of chronic obstructive pulmonary disease: when are antibiotics indicated? A systematic review. Respir Res 2007; 8: 30. doi: 10.1186/ 1465-9921-8-30.

35. Wedzicha JA. Role of viruses in exacerbations of chronic obstructive pulmonary disease. Proc Am Thorac Soc 2004; 1: 115-20.

36. Seemungal TA, Donaldson GC, Bhowmik A, Jeffries DJ, Wedzicha JA. Time course and recovery of exacerbations in patients with chronic obstructive pulmonary disease. Am J Respir Crit Care Med 2000; 161: 1608-13.

37. Seemungal T, Harper-Owen R, Bhowmik A, Moric I, Sanderson G, Message S, et al. Respiratory viruses, symptoms, and inflammatory markers in acute exacerbations and stable chronic obstructive pulmonary disease. Am J Respir Crit Care Med 2001; 164: 1618-23.

38. Papi A, Johnston SL. Rhinovirus infection induces expression of its own receptor intercellular adhesion molecule 1 (ICAM-1) via increased NF-kappaB-mediated transcription. J Biol Chem 1999; 274: 9707-20.

39. Seemungal TA, Harper-Owen R, Bhowmik A, Jeffries DJ, Wedzicha JA. Detection of rhinovirus in induced sputum at exacerbation of chronic obstructive pulmonary disease. Eur Respir J 2000; 16: 677-83.

40. Wilkinson TM, Hurst JR, Perera WR, Wilks M, Donaldson GC, Wedzicha JA. Effect of interactions between lower airway bacterial and rhinoviral infection in exacerbations of COPD. Chest 2006; 129: 317-24.

41. Barnes PJ, Celli BR. Systemic manifestations and comorbidities of COPD. Eur Respir J 2009; 33: 1165-85.

42. Bhowmik A, Seemungal TA, Sapsford RJ, Wedzicha JA. Relation of sputum inflammatory markers to symptoms and lung function changes in COPD exacerbations. Thorax 2000; 55: 114-20.

43. Wedzicha JA, Seemungal TA, MacCallum PK, Paul EA, Donaldson GC, Bhowmik A, et al. Acute exacerbations of chronic obstructive pulmonary disease are accompanied by elevations of plasma fibrinogen and serum IL- 6 levels. Thromb Haemost 2000; 84: 210-5.

44. Hurst JR, Donaldson GC, Perera WR, Wilkinson TM, Bilello JA, Hagan GW, et al. Use of plasma biomarkers at exacerbation of chronic obstructive pulmonary disease. Am J Respir Crit Care Med 2006; 174: 867-74.

45. Perera WR, Hurst JR, Wilkinson TM, Sapsford RJ, Müllerova H, Donaldson GC, et al. Inflammatory changes, recovery and recurrence at COPD exacerbation. Eur Respir J 2007; 29: 527-34.

46. Groenewegen KH, Postma DS, Hop WC, Wielders PL, Schlösser NJ, Wouters EF, Cosmic Study Group. Increased systemic inflammation is a risk factor for COPD exacerbations. Chest 2008; 133: 350-7.

47. Bathoorn E, Liesker JJ, Postma DS, Köeter GH, Van Der Toorn M, Van Der Heide S, et al. Change in inflammation in out-patient COPD patients from stable phase to a subsequent exacerbation. Int J Chron Obstruct Pulmon Dis 2009; 4: 101-9. 